POS $\quad$ PROCEEDINGS

\title{
Causal structure for noncommutative geometry
}

\author{
Michał Eckstein*† \\ Faculty of Physics, Astronomy and Applied Computer Science, Jagiellonian University, ul. prof. \\ Stanistawa Łojasiewicza 11, 30-348 Kraków, Poland \\ Faculty of Mathematics and Computer Science, Jagellonian University, ul. Łojasiewicza 6, \\ 30-348 Kraków, Poland \\ Copernicus Center for Interdisciplinary Studies, ul. Stawkowska 17, 31-016 Kraków, Poland \\ E-mail: michaldeckstein.pl, michal.eckstein@uj.edu.pl
}

Nicolas Franco,

Faculty of Mathematics and Computer Science, Jagellonian University, ul. Łojasiewicza 6, 30-348 Kraków, Poland

E-mail: nicolas.franco@im.uj.edu.pl, nicolas.franco@math.unamur.be

\begin{abstract}
Noncommutative geometry à la Connes offers a promising framework for models of fundamental interactions. To guarantee the correct signature, the theory of Lorentzian spectral triples has been developed. We will briefly summarise its main elements and show that it can accommodate a sensible notion of causality understood as a partial order relation on the space of states on a $C^{*}$-algebra. For almost commutative algebras of the form $C_{c}^{\infty}(M) \otimes A_{F}$, with $A_{F}$ being finitedimensional, the space of pure states is a Cartesian product of the space-time $M$ and an internal, 'quantum', space. The exploration of causal structures in this context led to a surprising conclusion: The motion in both space-time and the internal space is restricted by a 'finite speed of light' constraint. We will present this phenomenon on 2 simple toy-models.
\end{abstract}

Frontiers of Fundamental Physics 14 - FFP14,

15-18 July 2014

Aix Marseille University (AMU) Saint-Charles Campus, Marseille

\footnotetext{
* Speaker.

${ }^{\dagger}$ Supported within the project NCN PRELUDIUM 2013/09/N/ST1/01108.

${ }^{\ddagger}$ Supported within the project NCN PRELUDIUM 2013/09/N/ST1/01108.
} 


\section{Noncommutative geometry and Lorentzian signature}

The noncommutative geometry à la Connes is an algebraisation of the classical notion of Riemannian geometry which opens new horizons in mathematics - with the definition of 'noncommutative spaces', as well as in physics - with the construction of the noncommutative Standard Model coupled to Euclidean gravity $[1,2]$. However the basic object of noncommutative geometry a spectral triple $(A, H, D)$ - describes a noncommutative Riemannian space, whereas relativistic physics requires a Lorentzian signature. Some work in the direction of Lorentzian noncommutative geometry was carried out in $[3,4,5,6,7,8,9]$.

To extend the notion of spectral triple to the Lorentzian framework we first need the following analogue of a Hilbert space with an indefinite inner product [10, Chapter V]:

Definition 1. A (complex) vector space $K$ equipped with a non-degenerate inner product $(\cdot, \cdot): K \times$ $K \rightarrow \mathbb{R}$ is called a Krein space if there exists a decomposition $K=K^{+} \oplus K^{-}$, such that $\left(K^{+},(\cdot, \cdot)\right)$ and $\left(K^{-},-(\cdot, \cdot)\right)$ are Hilbert spaces and $\left(K^{+}, K^{-}\right)=\{0\}$.

With every orthogonal decomposition $K=K^{+} \oplus K^{-}$one can associate a bounded linear operator $\mathscr{J}: K \rightarrow K$ - the fundamental symmetry — by setting $\mathscr{J}=\mathrm{id}_{K^{+}} \oplus\left(-\mathrm{id}_{K^{-}}\right)$. Having chosen a $\mathscr{J}$, one can equip $K$ with a positive-definite inner product $\langle\cdot, \cdot\rangle:=(\cdot, \mathscr{J} \cdot)$ and thus turn $K$ into a Hilbert space. Let us stress however that the decomposition $K=K^{+} \oplus K^{-}$is not unique in general.

Whereas usually the definition of a pseudo-Riemannian spectral triple involves a Hilbert space and an operator $\mathscr{J}[4,6,8,9]$, we shall follow the original idea of [7] and work directly with a Krein space. This will assure that our definition of a causal structure does not depend on the choice of the decomposition.

Definition 2. A Lorentzian spectral triple $(A, K, D)$ consists of an involutive algebra $A$, a Krein space $K$ and a densely defined operator $D$ acting on $K$, such that:

1. $A$ is a dense $*$-subalgebra of a $C^{*}$-algebra $\bar{A}$.

2. There exists a faithful representation $\pi$ of $A$ as bounded operators on $K^{1}$.

3. $i D$ is Krein-symmetric, i.e. symmetric with respect to the indefinite inner product in $K$.

4. For all $a \in A$, the operator $[D, a]$ extends to a bounded operator on $K$.

5. The fundamental symmetry of $K$ commutes with the algebra $A$ and captures the Lorentzian signature.

A Lorentzian spectral triple is even if there exists a bounded operator $\gamma$ on $K$, such that $i \gamma$ is Kreinselfadjoint, $\gamma^{2}=1, \gamma D=-D \gamma$ and $[\gamma, a]=0 \forall a \in A$.

Let us note that without the, somewhat sloppy, condition (5) we would in general obtain a pseudo-Riemannian spectral triple. On the other hand, if we find one operator $\mathscr{J}$ fulfilling condition (5), it will be satisfied for any other fundamental symmetry. For a more explicit construction of $\mathscr{J}$ based on the existence of time functions on globally hyperbolic space-times see [11].

\footnotetext{
${ }^{1}$ By a standard abuse of notation, the symbol $\pi$ will be omitted.
} 
Example 3. Let $M$ be a smooth Lorentzian orientable and time-orientable spin manifold, then $\left(A_{M}, K_{M}, \not D\right)$ is a pseudo-Riemannian spectral triple, with $A_{M}=C_{c}^{\infty}(M), K_{M}=L^{2}(M, S)-$ the space of square summable sections of a spinor bundle $S$ over $M-$ and $\not D=-i e_{a}{ }^{\mu} \gamma^{a} \nabla_{\mu}^{S}-$ the standard Dirac operator $\left(\nabla^{S}\right.$ stands for the spin connection, $e_{a}{ }^{\mu}-$ the vielbeins and $\gamma^{a}-$ the flat gamma matrices) ${ }^{2}$. If $n$ is even, the $\mathbb{Z}_{2}$-grading is given by: $\gamma= \pm i^{\frac{n}{2}+1} \gamma^{0} \cdots \gamma^{n-1}$.

The space of sections of a spinor bundle over $M$ is indeed naturally equipped with an indefinite inner product $(f, g)=\int_{M}\left(f_{x}, g_{x}\right)_{x} \sqrt{|g|} d^{n} x$, which gives rise to the Krein space $K_{M}$. It has a canonical fundamental symmetry operator $\mathscr{J}_{M}=i \gamma^{0}$, which defines a positive inner product $\langle f, g\rangle=\int_{M}\left\langle f_{x}, g_{x}\right\rangle_{x} \sqrt{|g|} d^{n} x$, with $\langle\cdot, \cdot\rangle_{x}=\left(\cdot, i \gamma^{0} \cdot\right)_{x}$. This observation provides a mathematical explanation of the fact that the spinor adjoint to $\psi$ is $\bar{\psi}=\psi^{\dagger} \gamma^{0}$, rather than $\psi^{\dagger}$.

If the manifold $M$ is complete ${ }^{3}$, then the Dirac operator $i \not D$ is essentially Krein-selfadjoint [12], otherwise it is only Krein-symmetric [7].

Example 4. Let $\left(A_{M}, K_{M}, \not D\right)$ be an even spectral triple defined as in Example 3 and let $\left(A_{F}, H_{F}, D_{F}\right)$ be a finite (i.e. $A_{F}$ is finite dimensional) Riemannian spectral triple. Then, the data $A=A_{M} \otimes A_{F}$, $K=K_{M} \otimes H_{F}, D=\not D \otimes 1+\gamma_{M} \otimes D_{F}$ form an almost commutative Lorentzian spectral triple [13].

Note that there is a canonical Krein-space structure on $K_{M} \otimes H_{F}$ given by the product $((\cdot, \cdot))$ defined as $\left(\left(\psi \otimes e_{i}, \psi^{\prime} \otimes e_{j}\right)\right):=\left(\psi, \psi^{\prime}\right) \cdot \delta_{i, j}$, for $e_{i}$ - elements of the basis of $H_{F}$ - and extended by linearity.

\section{Causal structures in the space of states}

Recall that a state on $A$ is a positive linear functional of norm 1 . The set of states on $A$, denoted by $S(A)$, is a convex set (for the weak-* topology). Extremal points of $S(A)$ are called pure states and denoted by $P(A)$. If $A$ is a dense $*$-subalgebra of a $C^{*}$ algebra $\bar{A}$ one defines $S(A)$ and $P(A)$ by taking restrictions of the (pure) states on $\bar{A}$ to $A$.

Example 5. Let $M$ be a locally compact Hausdorff space and let $A_{M}=C_{0}^{\infty}(M)$ be the space of smooth functions on $M$ vanishing at infinity, then $P\left(A_{M}\right) \simeq M$ by the famous Gelfand-Naimark theorem. Pure states $\left\{\phi_{p}\right\}_{p \in M}$ on $A_{M}$ are given by the evaluation functionals, i.e. $\phi_{p}(a)=a(p)$ for all $a \in A_{M}$. More general states from $S\left(A_{M}\right)$ correspond to probability measures on $M$.

Example 6. Let $A_{F}=M_{n}(\mathbb{C})$ for some $n \in \mathbb{N}$. Then, every pure state on $A_{F}$ is a vector state, i.e. it is of the form $\phi(\cdot)=\langle\psi, \cdot \psi\rangle$, for some $\psi \in H_{F}=\mathbb{C}^{n}$. Since two vectors in $H_{F}$ differing by a phase factor yield the same state, we have $P\left(A_{F}\right) \simeq \mathbb{C} P^{n-1}$, known under the name of 'qunit' or ' $n$-qubit' in the quantum information theory [14]. General states on $A_{F}$ correspond to the density matrices.

Let $A$ be a tensor product of two (represented) $C^{*}$-algberas $A_{1}$ and $A_{2}$. A state on $A$ is called separable if it can be written as simple tensor $\phi \otimes \chi$ with $\phi \in A_{1}, \chi \in A_{2}$. If at least one of the algebras $A_{i}$ is commutative, then every pure state on $A_{1} \otimes A_{2}$ is separable [15, Theorem 11.3.7].

\footnotetext{
${ }^{2}$ Conventions used in the paper are $(-,+,+,+, \cdots)$ for the signature of the metric and $\left\{\gamma^{a}, \gamma^{b}\right\}=2 \eta^{a b}$ for the flat gamma matrices, with $\gamma^{0}$ anti-Hermitian and $\gamma^{a}$ Hermitian for $a>0$.

${ }^{3}$ I.e., there exists a spacelike reflection - an automorphism $r$ of the tangent bundle respecting $r^{2}=1, g(r \cdot, r \cdot)=$ $g(\cdot, \cdot)$ — such that $M$ equipped with the Riemannian metric $g^{r}(\cdot, \cdot)=g(\cdot, r \cdot)$ is complete in the Lebesgue sense.
} 
Thus, the space of pure states of an almost commutative Lorentzian spectral triple can be given a clear physical interpretation of being a Cartesian product of the space-time $M$ and an internal quantum - space of states of the model. General mixed states in $S(A)$ can entangle the space-time and inner components and their physical interpretation is not clear.

In [5] we proposed the following definition of causality for Lorentzian spectral triples:

Definition 7. Let $(A, K, D)$ be a Lorentzian spectral triple and let $\widetilde{A}$ be a unitisation of $A$, such that $\forall a \in \widetilde{A},[D, a]$ extends to a bounded operator on $K$ and $A$ is an ideal of $\widetilde{A}^{4}$. Let $\mathscr{C}$ be the cone of all Hermitian elements $a \in \widetilde{A}$ respecting

$$
\forall \phi \in K, \quad(\phi,[D, a] \phi) \leq 0 .
$$

If

$$
\overline{\operatorname{span}_{\mathbb{C}}(\mathscr{C})}=\overline{\widetilde{A}},
$$

where $\overline{\widetilde{A}}$ denotes the $C^{*}$-completion of $\widetilde{A}$, then $\mathscr{C}$ is called a causal cone. It defines a partial order relation on $S(\widetilde{A})$ (and a fortiori on $P(\widetilde{A})$ ), called the causal relation, by

$$
\forall \omega, \eta \in S(\widetilde{A}), \quad \omega \preceq \eta \quad \text { iff } \quad \forall a \in \mathscr{C} \quad \omega(a) \leq \eta(a) .
$$

The role of the condition (2.2) is to avoid acausal spaces. Indeed, if $M$ contains closed causal curves, the only functions in the causal cone are the constant ones and (2.2) fails [5].

To assure that the physical implications of the presented structure do not depend on the chosen unitisation (compare footnote ${ }^{4}$ ) we define the following space of physical pure states:

$$
\mathscr{M}(A):=\{\omega \in P(\widetilde{A}): A \not \subset \operatorname{ker} \omega\} \cong P(A) .
$$

Definition 7 is strongly motivated by the following result [5, Theorem 7].

Theorem 8. Let $\left(A_{M}, K_{M}, \not D\right)$ be a Lorentzian spectral triple constructed from a complete globally hyperbolic manifold $M$. Two physical pure states $\phi_{p}, \phi_{q} \in M\left(A_{M}\right)$ are causally related with $\phi_{p} \preceq \phi_{q}$ if and only if $p \preceq q$ in $M$.

Our approach to characterize the causal structure of a noncommutative space is in some points similar to the approach of Fabien Besnard [16] also presented at this conference. The main difference is that our construction is directly related to the Dirac operator, hence to the 'metric', and always corresponds to the usual causal structure in the commutative case, while the approach of Fabien Besnard may include more general partial orders.

\section{Testing the concepts - almost commutative causality}

Theorem 9. Let $(A, K, D)$ be an almost commutative Lorentzian spectral triple, such that the causal cone $\mathscr{C}$ exists. If $\omega_{p, \xi}, \omega_{q, \chi} \in \mathscr{M}(A)$ are such that $\omega_{p, \xi} \preceq \omega_{q, \chi}$, then $p \preceq q$ in $M$.

\footnotetext{
${ }^{4}$ The role of the unitisation is only technical and has no impact on the definition of causality for physical states, at least in the almost commutative case. For a more detailed discussion of that point, we refer the reader to [13].
} 
Proof. First note that $a \otimes 1$, with a smooth causal function $a$ is always in the causal cone $\mathscr{C}$, since $[D, a \otimes 1]=[\not D, a]$. Secondly, since pure states on $A_{F}$ are always vector states we have $\omega_{p, \xi}(a \otimes 1) \leq \omega_{q, \chi}(a \otimes 1) \Leftrightarrow\langle\xi, \xi\rangle a(p) \leq\langle\chi, \chi\rangle a(q) \Leftrightarrow a(p) \leq a(q) \Rightarrow p \preceq q$.

This result implies that whenever an almost commutative Lorentzian spectral triple admits a causal structure, no evolution of the physical system modelled by it induces faster-than-light effects.

Using the construction of an almost commutative spectral triple (4) with the two-point space $A_{F}=\mathbb{C} \oplus \mathbb{C}, H_{F}=\mathbb{C}^{2}$ and $D_{F}=\left(\begin{array}{cc}0 & m \\ m & 0\end{array}\right)$, with $m \in \mathbb{C}$, we obtain a two-sheeted space-time. The space of pure states on $A=A_{M} \otimes A_{F}$ consists of two copies of the manifold $M$, but its geometry is noncommutative as $D_{F}$ does not commute with the algebra. The following result is proved in [17] for dimensions 2 and 4 :

Theorem 10. Two pure states corresponding respectively to the event $p$ on the first sheet and an event $q^{\prime}$ on the second sheet are causally related if and only if $p \preceq q$ on $M$ (where $q$ is the corresponding point of $q^{\prime}$ on the first sheet - see Figure 1) and

$$
l(\gamma) \geq \frac{\pi}{2|m|},
$$

where $l(\gamma)$ represents the length of a causal curve $\gamma$ going from $p$ to $q$ on the first sheet.

This result, illustrated on Figure 1, implies that causal relations between a priori disconnected sheets are possible. We note that the Riemannian distance between the two sheets (obtained using Connes' distance formula [1]) is $d=\frac{1}{|m|}$.

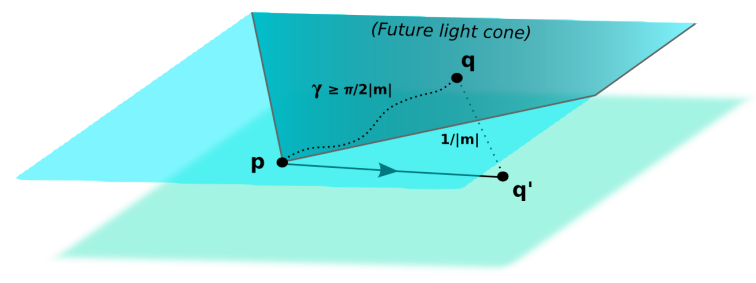

Figure 1: Causal structure for the two-sheeted model.

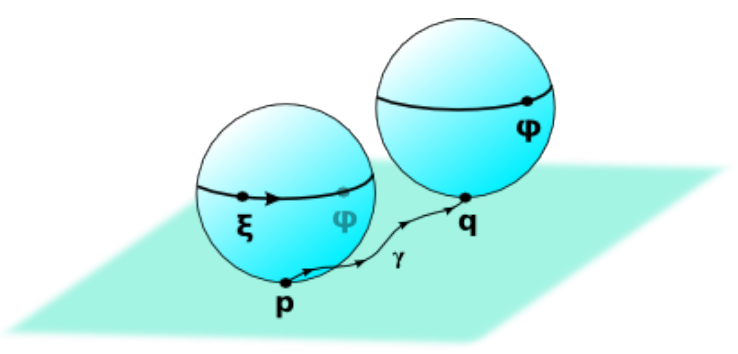

Another almost commutative model can be built using the following finite spectral triple: $A_{F}=M_{2}(\mathbb{C}), H_{F}=\mathbb{C}^{2}$ and $D_{F}=\left(\begin{array}{ll}d_{1} & 0 \\ 0 & d_{2}\end{array}\right)$, with $d_{1}, d_{2} \in \mathbb{R}$. The internal space corresponds to $\mathbb{C} P^{1} \cong S^{2}$ and a pure state $\omega_{p, \xi}$ on the product spectral triple is uniquely specified by $p \in M$ and $\xi \in S^{2}$. The following theorem holds with $M$ being the 2-dimensional Minkowski space-time [13]:

Theorem 11. Two pure states $\omega_{p, \xi}, \omega_{q, \varphi}$ are causally related with $\omega_{p, \xi} \preceq \omega_{q, \varphi}$ if and only if the following conditions are respected:

- $p \preceq q$ in $\mathbb{R}^{1,1}$;

- $\xi$ and $\varphi$ have the same latitude (they belong to the same parallel of latitude on $S^{2}$ );

- $l(\gamma) \geq \frac{\left|\theta_{\varphi}-\theta_{\xi}\right|}{\left|d_{1}-d_{2}\right|}$, where $l(\gamma)$ represents the length of a causal curve $\gamma$ going from $p$ to $q$ on $\mathbb{R}^{1,1}$ and $\theta_{\xi}, \theta_{\varphi}$ are the angles of $\xi, \varphi$ on the parallel of latitude. 
This result is illustrated by Figure 2. The constraint $l(\gamma) \geq \frac{\left|\theta_{\varphi}-\theta_{\xi}\right|}{\left|d_{1}-d_{2}\right|}$ implies that the proper time on the space-time must be sufficient in order to allow any motion within the internal space. This means that the ratio of the distance in the internal space to the proper time $\frac{\left|\theta_{\varphi}-\theta_{\xi}\right|}{l(\gamma)}$ is bounded above by the constant $\left|d_{1}-d_{2}\right|$, which can be seen as a speed of light limit existing within the internal space.

\section{References}

[1] A. Connes, Noncommutative Geometry. Academic Press, 1994.

[2] A. Connes and M. Marcolli, Noncommutative Geometry, Quantum Fields and Motives, vol. 55 of Colloquium Publications. American Mathematical Society, 2008.

[3] P. Bieliavsky, S. Detournay, P. Spindel, and M. Rooman, Star products on extended massive non-rotating BTZ black holes, Journal of High Energy Physics 06 (2004), no. 031.

[4] N. Franco, Temporal Lorentzian spectral triples, Reviews in Mathematical Physics 26 (2014), no. 08 1430007, [arXiv:1210.6575].

[5] N. Franco and M. Eckstein, An algebraic formulation of causality for noncommutative geometry, Classical and Quantum Gravity 30 (2013), no. 13 135007, [arXiv: 1212. 5171].

[6] M. Paschke and A. Sitarz, Equivariant Lorentzian spectral triples, (2006) [math-ph/0611029].

[7] A. Strohmaier, On noncommutative and pseudo-Riemannian geometry, Journal of Geometry and Physics 56 (2006), no. 2 175-195, [math-ph/ 0110001 ].

[8] K. van den Dungen, M. Paschke, and A. Rennie, Pseudo-Riemannian spectral triples and the harmonic oscillator, Journal of Geometry and Physics 73 (2013) 37-55, [arXiv:1207.2112].

[9] R. Verch, Quantum Dirac field on Moyal-Minkowski spacetime - illustrating quantum field theory over Lorentzian spectral geometry, in Acta Physica Polonica B Proceedings Supplement, vol. 4, pp. 507-530, 2011. arXiv:1106.1138.

[10] J. Bognár, Indefinite Inner Product Spaces. Springer, 1974.

[11] N. Franco and M. Eckstein, Noncommutative geometry, Lorentzian structures and causality, in Mathematical Structures of the Universe (M. Eckstein, M. Heller, and S. Szybka, eds.), pp. 315-340. Copernicus Center Press, 2014. arXiv: 1409.1480.

[12] H. Baum, Spin-Strukturen und Dirac-Operatoren über pseudoriemannschen Mannigfaltigkeiten, vol. 41 of Teubner-Texte zur Mathematik. Teubner, 1981.

[13] N. Franco and M. Eckstein, Exploring the causal structures of almost commutative geometries, Symmetry, Integrability and Geometry: Methods and Applications 10 (2014), no. 010 [arXiv:1310.8225]. Special Issue on Noncommutative Geometry and Quantum Groups.

[14] I. Bengtsson and K. Życzkowski, Geometry of Quantum States: an Introduction to Quantum Entanglement. Cambridge University Press, 2006.

[15] R. V. Kadison and J. R. Ringrose, Fundamentals of the Theory of Operator Algebras, vol. 2. Advanced theory. Academic Press, 1986.

[16] F. Besnard, Causality and noncommutative geometry, in Frontiers of Fundamental Physics 14, 2014. PoS(FFP14)132.

[17] N. Franco and M. Eckstein, Causality in noncommutative two-sheeted space-times, (2015). arXiv: 1502.04683. 